\title{
Espacio y tiempo en las representaciones infantiles de la muerte***
}

\author{
Space and time in children's representations of death
}

\section{RESUMEN}

En base a los resultados obtenidos en una investigación empírica sobre el desarrollo del conocimiento infantil acerca de la muerte humana (realizada con niños argentinos de 5 a 10 años de edad, pertenecientes a sectores socioculturales medios y a grupos de familias religiosas y ateas), se presenta en este artículo una discusión teórica acerca de dos aspectos centrales de la comprensión de la muerte: lo que denominamos espacialización (la caracterización de la muerte, no en los términos de los procesos biológicos o de las representaciones religiosas usuales, sino de un lugar o espacio) y la secuencia temporal (la organización temporal que adoptan los hechos en las explicaciones, motivaciones y búsqueda de razones de la muerte). A modo de hipótesis, se propone que son estos aspectos del desarrollo de la comprensión de la muerte en los niños los que dan lugar a las nociones polifásicas de las representaciones, creencias y prácticas de los adultos.

Palabras Clave: Representaciones infantiles de la muerte - Desarrollo cognitivo - Muerte e infancia - Espacialización - Secuencia temporal.

\begin{abstract}
Based on the results obtained in an empirical study on the development of children's knowledge on human death (conducted with Argentinian children from the ages of 5 to 10, belonging to middle-class atheist and religious families), we present in this article a theoretical discussion regarding two central aspects in the comprehension of death: what we will call spatialization (the characterization of death, not in biological terms or in terms of the usual religious representations, but in terms of a place or space) and the temporal sequence (the temporal organization of facts, explanations, motivations and the search for reasons for death). As a hypothesis, we propose that these are the aspects, involved in the development of the understanding of death in children, that lead to adults' polyphasic notions of representations, beliefs and practices.
\end{abstract}

Keywords: Child representations of death - Cognitive development Death and Childhood - Spatialization - Temporal sequence.

* Profesor Adjunto de la Facultad de Psicología de la Universidad Nacional de La Plata, Argentina. CV: < http:// www.psico.unlp.edu.ar/inipsi>

** Profesora Titular (R) de la Facultad de Psicología de la Universidad Nacional de La Plata; Profesora Adjunta Profesora Adjunta(R) de la Facultad de Psicología de la Universidad de Buenos Aires, Argentina. CV: <http://www. psi.uba.ar/>.

*** Este artículo refiere a los resultados de una investigación doctoral sobre el desarrollo de la noción de muerte en los niños, financiada por el Consejo Nacional de Investigaciones Científicas y Técnicas de Argentina (CONICET). dirigida por Alicia María Lenzi y codirigida por José Antonio Castorina. 


\section{La muerte como objeto de conocimiento}

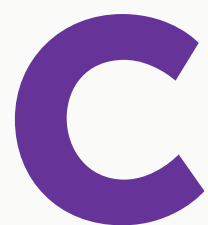

onstituiría un reduccionismo inadecuado postular un único evento o instante filogenético inaugural de lo humano, entre otras razones por las múltiples relaciones dialécticas entre una adquisición, sus resultados y los efectos de estos sobre la adquisición misma y las interacciones con otros (Tomasello, 2007). Sin embargo, la comprensión de la muerte es un tema tan central para nuestra especie que algunos autores han postulado que constituye uno de los aspectos fundamentales del proceso de hominización. Las pruebas paleontológicas capitales acerca de una posible conciencia sobre la muerte y otras creencias asociadas son las aparentes ofrendas y las disposiciones sistemáticas de los cuerpos (Arrizabalaga, 2010), vinculadas a la eclosión de una rudimentaria capacidad de simbolización (Binant, 1991; Defleur, 1993; Arrizabalaga, 2010). Mucho más recientemente, nuestra historia documentada no deja dudas acerca del lugar insistente y central que la conciencia de la muerte ha tenido para el hombre de todas las épocas. Desde las antiguas tablillas babilónicas sobre la Epopeya de Gilgamesh hasta nuestros días, una y otra vez la producción cultural de nuestra especie, en todas sus variantes, "niega y reconoce la muerte; la niega como paso a la nada; la reconoce como acontecimiento" (Morin, 1994, p. 24). La respuesta a la pregunta por el momento en el que la muerte adquirió tal relevancia para nuestra especie parece insondable. Ahora bien, ¿en qué momento de nuestro desarrollo cognitivo ontogenético esta conciencia de la muerte, tan velada como omnipresente en las prácticas humanas, adquiere su representación adulta? ¿De qué manera ocurre este desarrollo?

Entender el modo en el cual los niños adquieren ideas "adultas" sobre la muerte humana constituye un problema complejo. Por una parte, la muerte es un objeto de conocimiento heterogéneo y de difícil delimitación en términos de campos o dominios de experiencias específicas (Castorina y Faigenbaum, 2000; Rosengren, Gutiérrez y Schein, 2014). Supone un aspecto material biológico, porque refiere indefectiblemente a ciertos componentes y relaciones observables ${ }^{1}$ en un organismo vivo (Slaughter, Jaakola y Carey, 1999). En este terreno biológico es donde se sitúa el conocimiento acerca de las transformaciones que ocurren a lo largo del tiempo, así como la comprensión de la cesación de las funciones orgánicas (Inagaki y Hatano, 2006). Sin dudas, podemos afirmar que comprender la muerte implica reconocer la existencia de ciertos seres vivos que se desarrollan y que, en algún momento de ese desarrollo, cesan sus funciones vitales como consecuencia de alguna causa conocida o desconocida. Pero la comprensión de la muerte no se agota en el conocimiento de sus aspectos biológicos. Efectivamente, la muerte es, al mismo tiempo, un objeto de conocimiento social y cultural, porque las creencias, las prácticas y los valores compartidos y reproducidos por el grupo social de pertenencia constituyen otros de los tantos niveles inherentes al objeto, niveles que se encuentran densamente imbricados (Gire, 2014; Tau y Lenzi, 2015). Al respecto, debemos recordar que

1 La noción de "observable" no refiere aquí necesariamente a una actividad perceptiva, sino a lo que el sujeto cree comprobar en los hechos, siendo esta comprobación dependiente de sus instrumentos de registro -los esquemas asimilatorios y sus relaciones- disponibles en cada nivel del desarrollo del conocimiento (Piaget, 1998). 
desde la psicología genética piagetiana se ha teorizado profundamente sobre la relación entre el sujeto y el objeto de conocimiento. Desde esta perspectiva -a la que nos referiremos más adelante-, la complejidad de un objeto de conocimiento es simétrica respecto de la complejidad de los esquemas asimilatorios. Ya en el temprano desarrollo sensoriomotor del niño las experiencias visuales y motrices, por ejemplo, no refieren a un mismo objeto sino hasta el momento en el que los esquemas implicados en estas actividades se asimilan de manera recíproca (García, 2000; Piaget, 1936). Sin embargo, el objeto no es una creación autística del sujeto y la acomodación es un testimonio de la existencia de un más allá de la experiencia del sujeto, algo que recuerda al noúmeno kantiano (Pfeiffle, 2008).

En ciertos tipos de objetos de conocimiento, o dominios, existen propiedades y relaciones que están facilitadas o restringidas, algo que también da cuenta de ese más allá de la experiencia. En los objetos físicos estas propiedades son, comparativamente, muy estables. En el caso de la noción de muerte, diferentes dominios se entrecruzan. Allí, por ejemplo, el conocimiento biológico está interferido por la ideología -y viceversa- y cada uno de estos dominios le ofrece recursos semióticos al otro, aunque los campos de conocimientos se mantengan relativamente autónomos a lo largo del desarrollo sin que se elimine completamente la contradicción o los desequilibrios que la producen. Esta complejidad distintiva del objeto es algo que aquí solo enunciamos y no podemos desarrollar, aunque es coherente con la tesis acerca de la especificidad que el tipo de objeto le confiere al desarrollo de su conocimiento.

Es cierto que, en sentido estricto, todos los objetos de conocimiento -aun aquellos propios de la experiencia física o logicomatemática -están cargados de valores y usos sociales específicos. Sin embargo, en el caso de la muerte cada uno de sus aspectos nos remite a los otros, resultando difícil operar el seccionamiento teórico y metodológico que en otros dominios resulta efectivo para la definición clara de los problemas.

Al caracterizar un objeto como "social" queremos señalar, además de su existencia en una trama de sentidos socialmente construida, el hecho de que el mismo sujeto forma parte de las prácticas sociales en las que el objeto alcanza su consistencia o estatuto ontológico. El sujeto cognoscente es un actor social (Psaltis, Duveen y Perret-Clermont, 2009) y sus experiencias se sitúan siempre en el seno de instituciones en las que las acciones cobran significado (Castorina, Clemente y Barreiro, 2005; Castorina, Kohen Kohen y Zerbino, 2000). Podemos decir, sintéticamente, que "un objeto social coacciona al sujeto, porque lo coloca en determinado lugar institucional, lo disciplina o le prescribe actos que debe cumplir" (Lenzi y Castorina, 2000, p. 44). De este modo, las interacciones socialmente reguladas modulan el desarrollo del conocimiento individual, orientándolo hacia una cosmovisión compartida, a través de los intercambios con los otros, las prácticas instituidas y los recursos semióticos disponibles en los diferentes niveles contextuales (Valsiner, 2005; 2012; Valsiner y Winegar, 1992). Esto no significa que el conocimiento individual sea el efecto de una recepción pasiva ni que se explique apelando a tesis ambientales de inculcación. Ya en una de sus obras más tempranas sobre el desarrollo del conocimiento, Piaget (1926) mostró de qué manera los niños, en lugar de copiar o imitar pasivamente las ideas de los adultos, se apropian de las representaciones del mundo 
que pertenecen a su contexto social a través de una reelaboración original de las creencias o nociones que circulan en el grupo de pertenencia. En el mismo sentido, se puede sostener que los significados institucionalizados son siempre fenómenos de interface: 2

en el funcionamiento de toda institución social es inútil el querer señalar la parte del individuo y la parte social; el proceso social y su influjo en las consciencias individuales son una sola y misma cosa, o más exactamente, constituyen las dos fases de una misma realidad. (Piaget, 1975, p. 331).

Pero este desarrollo del conocimiento no sucede ex nihilo, sino como consecuencia de las interacciones con determinados objetos situados en una cosmovisión o marco epistémico particular (García, 2001).

Es así que, comprender la muerte, no es sólo volver gradualmente inteligible al fenómeno biológico de la cesación de las funciones vitales, sino también apropiarse del conjunto de prácticas, creencias y valores del grupo de pertenencia -apropiación ideológica que es incluso anterior o relativamente independiente respecto del desarrollo nocional o conceptual correspondiente, como han mostrado diversas investigaciones análogas (Lloyd y Duveen, 1990; Tajfel, 1981)-. Es en este sentido que sostenemos que el desarrollo de la comprensión de la muerte es tanto un conocimiento sobre la sociedad y la cultura en sus diferentes niveles -es decir, particular o contextual- como un conocimiento sobre procesos e invariantes biológicas - universal-. Se trata de un tipo de objeto en el que se intersectan conocimientos físicos, biológicos, logicomatemáticos y sociales. Adicionalmente, los sentidos instituidos, las creencias, opiniones y otras formas de la ideología del grupo son altamente inestables y mutables, mientras que los aspectos biológicos, por su parte, ofrecen una mayor fijeza, una "sustantividad en cuanto al cumplimiento de una transformación" (Castorina et al., 1989) y una consecuente mayor predictibilidad, propia de los objetos naturales. Para indagar la participación de estos diferentes niveles contextuales en el pensamiento infantil son necesarias múltiples estrategias de coordinación teórica y metodológica -siendo el análisis de los sistemas de creencias familiares especialmente relevante al momento de conjeturar una primera relación entre el desarrollo del conocimiento del niño y los contextos (Lightfoot y Valsiner, 1992)-.

Tenemos, además, otra complejidad que debemos considerar: el conocimiento sobre la muerte no refiere al hipotético instante en el que la muerte ocurre sino a dos momentos que se suceden y conectan a través de un punto virtual de pasaje. Nos referimos, por un lado, al

2 Utilizaremos el vocablo interfaz, un anglicismo incorporado con un sentido restringido al Diccionario de la Real Academia Española - "conexión o frontera común entre dos aparatos o sistemas independientes" (Real Academia Española, 2013) - diferente del de su homófono americano interfase. Al respecto, recordamos que la voz inglesa interface "[...] se ha adaptado al español en la forma interfaz [...]. Su plural es interfaces. [...] Con este sentido no debe usarse la forma interfase, que no corresponde ni a la pronunciación ni a la estructura semántica del étimo inglés, que se ha formado con el sustantivo face, cuyo equivalente español es faz, no fase" (Academia Argentina de Letras, 2011, p. 216-217). Con todo, si bien la definición de interfaz ofrecida por la RAE se aproxima al sentido con el que la utilizamos aquí, la perspectiva dialéctica, desde la cual se concibe la vinculación entre las representaciones sociales y el conocimiento subjetivo se desvanece al postular una conexión entre "sistemas independientes". No obstante, mantendremos este término, precisando, en cada caso, un campo semántico que evite cualquier reminiscencia disyuntiva. 
momento en el que se pueden situar las razones de ocurrencia de la muerte (que denominamos MRO), tanto en términos causales como motivacionales. Por otro lado, al momento post mortem, referido al después o más allá de la muerte (MDM). En ambos momentos, anterior y posterior respecto de la muerte como pasaje o punto medio virtual, es posible reconocer una espacialidad y una temporalidad, dos ejes del desarrollo de la comprensión de la muerte humana de los que nos ocuparemos en este artículo (Ver figura 1).

Figura 1. Diacronía del conocimiento sobre la muerte

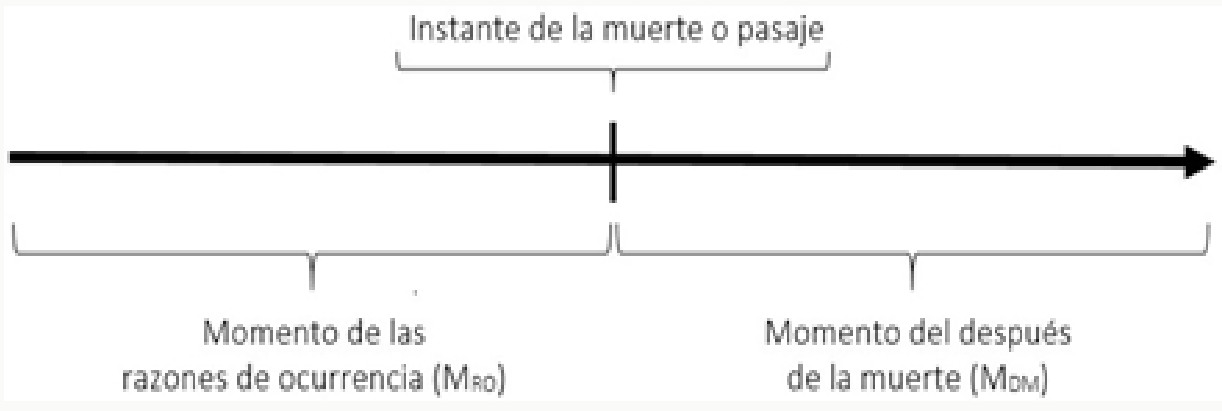

\section{Aspectos metodológicos de nuestra investigación}

El análisis teórico que presentamos en este escrito se basa en los resultados de una investigación empírica sobre el desarrollo de la comprensión infantil de la muerte humana, en consecuencia, es necesario mencionar sus aspectos generales, al menos de manera fragmentaria y sucinta, para clarificar el origen de los datos.

La perspectiva teórica asumida en la investigación fue la de la psicología genética piagetiana, caracterizada como "el estudio del desarrollo de las funciones mentales, en tanto este desarrollo puede ofrecer una explicación, o por lo menos una información complementaria de sus mecanismos [...]" (Piaget, 1979, p. 47). Dicha teoría se inscribe en el proyecto general de una epistemología genética abocada al estudio de "las etapas sucesivas de una ciencia S en función de su desarrollo3" (Piaget, 1957, p. 13). En acuerdo con la especificidad de los objetos de ambas disciplinas - psicología y epistemología genéticas- y de la relativa autonomía generada por ambos programas de investigaciones, es posible sostener la distinción, aunque ambas sean parte de una "teoría general del conocimiento" que asume la imposibilidad de escindir los mecanismos del desarrollo del conocimiento precientífico y científico (García, 2000). Esta perspectiva ha sido revisada y desarrollada en muchos aspectos y nosotros adoptamos una extensión "crítica" de ella (Castorina, 2010; Psaltis y Duveen, 2006), en la que se redefine

3 "L'épistémologie génétique est l'étude des états successifs d'une science $S$ en fonction de son développement" (traducción nuestra) 
el papel del contexto y de las interacciones con los otros, se introduce la noción de dominio y se establece una coordinación teórica con la teoría iniciada por Moscovici (1979) acerca del "conocimiento de sentido común" (Castorina, 2008). En efecto, las representaciones sociales moscovicianas configuran maneras "de interpretar y de pensar nuestra realidad cotidiana, una forma de conocimiento social" (Jodelet, 1986, p. 473). Se trata de conocimientos cotidianos, distantes del conocimiento científico, elaborados, compartidos y reproducidos socialmente por un grupo. Al hablar de representación, en términos generales, hacemos referencia a un fenómeno simbólico de sustitución de un elemento representado por su representante (Ibidem). Pero en el caso de la representación social, los elementos representados son siempre objetos de naturaleza social que poseen un carácter de imagen simbólica y significante, se construyen y son creativos.

Por otra parte, la muestra de nuestra investigación estuvo constituida por cuotas intencionales, es decir, mediante un método de muestreo no probabilístico -por lo tanto, no todos los individuos tuvieron la misma probabilidad de ser seleccionados para representar la población estudiada-. Los participantes fueron 60 niños (30 mujeres y 30 varones) de cinco a diez años de edad, distribuidos en cuotas de diez sujetos por cada uno de los años del rango establecido. Los niños fueron entrevistados siguiendo la dinámica general del llamado "método clínico-crítico" (Castorina, Lenzi y Fernández, 1984; Piaget, 1975; Tau y Gómez, 2016), redefinido en función de la temática y expandido mediante la utilización de una fase de producción gráfica. El guion básico de las interacciones entrevistador-entrevistado fue definido luego de cinco pruebas piloto que aquí no consideramos y que nos sirvieron para ajustar progresivamente el instrumento de indagación. El objetivo general fue, siempre, acceder al punto de vista del niño acerca de la muerte humana y, al respecto, este tipo de entrevista semidirigida evita las limitaciones propias de las pruebas estandarizadas (Honey, 1987). A su vez, se entrevistaron a los 60 padres de cada uno de los niños participantes, a fin de obtener información sobre las creencias familiares y las experiencias cercanas con la muerte. Todos los niños pertenecían al partido de La Plata, Provincia de Buenos Aires, Argentina, y asistían a escuelas de gestión pública (65\%) y de gestión privada (35\%). El $100 \%$ de los niños pertenecía a sectores socioculturales medios (sin Necesidades Básicas Insatisfechas ${ }^{4}$, padres alfabetizados, 5\% con educación primaria completa; $26,7 \%$ con educación secundaria completa y $68,3 \%$ con educación universitaria completa). Respecto de las creencias religiosas, el $40 \%$ de los niños pertenecía a familias creyentes de tradición judeocristiana, mientras que el $60 \%$ restante se descompone de la siguiente manera: el 26,7\% pertenecía a familias agnósticas/ateas; el 33,3\% a familias con "creencias contradictorias" (aquellas en las que sólo uno de los padres se define como creyente y el otro como ateo/agnóstico).

4 El concepto de Necesidades Básicas Insatisfechas (NBI) refiere a un método de identificación de carencias críticas en una población, muy utilizado en América Latina para la delimitación de grupos de pobreza estructural. Usualmente, y por recomendación de la Comisión Económica para América Latina y el Caribe (Cepal, organismo dependiente de la Organización de las Naciones Unidas), las necesidades consideradas son la vivienda, los servicios sanitarios básicos, el acceso a la educación y la capacidad económica para alcanzar niveles mínimos de consumo. 
Tabla 1. Composición de la muestra

\begin{tabular}{|c|c|c|c|c|c|c|c|}
\hline \multicolumn{7}{|c|}{ Muestra definitiva } \\
\hline \multicolumn{2}{|c|}{ Tipo de escuela } & $\begin{array}{r}\text { Máximo nivel educativo alcanzado por al } \\
\text { menos uno de los padres }\end{array}$ & \multicolumn{3}{c|}{ Creencias religiosas familiares } \\
\hline Pública & Privada & Primario & Secundario & Universitario & $\begin{array}{c}\text { Creyentes } \\
\text { (tradición } \\
\text { judeocristiana) }\end{array}$ & $\begin{array}{c}\text { Agnósticos/ } \\
\text { ateos }\end{array}$ & $\begin{array}{c}\text { Creencias } \\
\text { contradictorias }\end{array}$ \\
\hline $65 \%$ & $35 \%$ & $5 \%$ & $26,7 \%$ & $68,3 \%$ & $40 \%$ & $26,7 \%$ & $33,3 \% E$ \\
\hline
\end{tabular}

\section{Argumentaciones infantiles en torno al MRO y al MDM}

Los dos momentos a los que refiere la comprensión de la muerte, el de las razones de su ocurrencia (MRO) y el del más allá (MDM), definen en su encuentro un borde brumoso que denominamos instante o pasaje virtual de la muerte. Ambos suponen una diacronía, aunque, como veremos, la organización del tiempo no configura una secuencia lineal irreversible para los niños de todos los niveles. Sobre la totalidad de esta secuencia (MRO + MDM) las diferentes culturas han elaborado conocimientos, representaciones, ritos y creencias más o menos sistematizadas, con una distribución desigual del foco hacia cada uno de los dos momentos.

\section{Argumentos acerca de las razones de la ocurrencia de la muerte (MRO)}

En los grupos sociales a los que pertenece la muestra que hemos estudiado, sobre el MRO se han hallado, primordialmente, conocimientos sistematizados y estables que se corresponden con un tipo de observable biológico-corporal y con una racionalidad cercana a la del conocimiento científico. Dicho de otro modo, los niños, como los adultos, dan cuenta de las razones de ocurrencia de la muerte humana mediante un argumento que se apoya en los conocimientos del dominio biológico. Con todo, este campo de objetos rebasa los conocimientos científicos de la biología y está integrado por nociones precientíficas, de sentido común o "naíf", acerca del cuerpo y su funcionamiento. A este dominio pertenecen los observables del organismo humano, coordinados de manera más o menos sistemática en una trama de relaciones que dan cuenta de procesos, jerarquías, dependencias y vulnerabilidades del cuerpo. También a este MRO pertenecen los observables sobre la enfermedad, la vejez y los agentes traumáticos -físicos y mentales- que de algún modo participan en las explicaciones usuales de la muerte, que los niños evidencian. Desde luego que en el MRO también se pueden hallar razones y motivaciones alejadas del dominio de la biología en sentido amplio. El "destino", la 
"voluntad divina" o la "suerte" son algunas de las motivaciones -observables, en el sentido en el que utilizamos esta noción- que invocan los niños al momento de justificar o explicar una muerte ocurrida. Sin embargo, ninguna de estas motivaciones aparece completamente despojada o desvinculada de los observables biológicos del cuerpo cuando se responde al porqué de una muerte. De manera más precisa, lo que se advierte es que las motivaciones no biológicas parecen configurar un campo general de condiciones para que los aspectos biológicos de la muerte se manifiesten.

Este tipo de representaciones es evidente en los niños del límite etario superior de nuestra muestra, quienes suelen coordinar, tanto de manera "clisé" como original, los conocimientos sobre el cuerpo con las motivaciones de la religión o el orden del mundo que hace de la muerte algo inevitable y universal. Por ejemplo, Jorge $(10,0)^{5}$, está seguro de que todas las personas, tarde o temprano, se tienen que morir, y que eso ocurre finalmente por algún tipo de disfuncionalidad, enfermedad o traumatismo en el cuerpo, aunque la razón última de esta condición ineludible de todo ser vivo se deba a una voluntad: la de Dios. En contextos seculares, las ideas biológicas de los niños de la misma edad también se inscriben en representaciones más amplias acerca de las causas o motivaciones fundamentales de la ocurrencia de la muerte. La inevitabilidad de la muerte, reconocida sin titubeos, puede justificarse en estos casos mediante una concepción de la vida como desgaste gradual, en la que las capacidades y funciones necesariamente se van agotando, limitando o perdiendo. En otros niños, las razones de la inevitabilidad de la muerte se basan en una justicia del mundo que castiga las malas conductas -v. g., Pablo, 5,0: “Los nenes se pueden morir? -No, los ladrones. - ¿Los ladrones? -Sí, porque ellos son los malos"6 - o en la predestinación de un tiempo limitado para un ser vivo -V. g., Sara, 8,2: "-¿La persona puede vivir miles de años? -No. - ¿Por qué? -Por la edad podés morir también. Si llegás a los cien años no podés seguir. - ¿No podés seguir? ¿Y si no tenés una enfermedad o no te pisa una moto, como me dijiste? -No, podés morir también si sos muy viejo. Te vas achicando" $-{ }^{7}$. Independientemente de la forma particular adoptada al justificar la necesidad de la muerte, subrayamos la relación que estas representaciones sobre el mundo mantienen con los conocimientos biológicos.

Del mismo modo, en las argumentaciones rudimentarias de los niños más pequeños, se distinguen observables del cuerpo relacionados de manera inestable con el campo de las motivaciones que no pertenecen al dominio biológico sino a la imaginería religiosa y a las representaciones difundidas en cuentos, películas, dibujos animados, entre otros. Así, en los niños de cinco años de edad aproximadamente, hallamos argumentos acerca de las razones de ocurrencia de la muerte con este tipo de entrecruzamientos entre el dominio biológico y las motivaciones no biológicas, aunque de forma mucho menos diferenciada que en los niños mayores. Por un lado, en estos casos predominan los observables sobre la sangre y el corazón, dos elementos que se mencionan frecuentemente para explicar las muertes: por cortes,

5 Utilizamos esta notación para indicar la edad de los niños de la muestra: (años, meses).

6 Para citar fragmentos de entrevistas utilizaremos tipografía cursiva para las intervenciones del entrevistador y regular para las del entrevistado.

7 Entrevistas clínico-críticas administradas en la ciudad de La Plata, Argentina, en agosto de 2012. 
disparos y diversos traumas físicos dejan escapar la sangre del cuerpo-saco o mediante la apelación a la ausencia de latidos que indican que la persona está muerta. De manera todavía menos diferenciada, algunos niños pequeños toman al cuerpo como un único observable sin distinciones claras de partes y funciones. Así, la causa de la muerte se debe a una súbita desaparición de la persona entera -v. g., "no la vemos más", "vamos a su casa y ya no está", "no atiende el teléfono", etc. $-{ }^{8}$. Al mismo tiempo, también junto a estos observables biológicos poco diferenciados, estos niños introducen en sus argumentaciones motivaciones de las que no participan los observables sobre el cuerpo, como son la causalidad moral de la muerte o la voluntad de Dios.

Se concluye entonces, que al referirse al MRO, el niño de diferentes niveles de desarrollo apela a motivaciones y observables no biológicos, asimilándolos a los esquemas de conocimientos biológicos o viceversa. Estas asimilaciones recíprocas e inestables entre dominios de experiencias diferentes dan lugar a juicios, correspondencias, explicaciones y pseudo explicaciones poco sistematizadas, circulares o, por momentos, contradictorias.

Dicha situación no parece ser exclusiva del pensamiento infantil. En los contextos sociales en los que las creencias religiosas constituyen una parte central del marco epistémico de los adultos (Piaget y García, 1982), el conocimiento sobre el cuerpo y las prácticas médicas correspondientes no se minimizan, sino que coexisten. Así, para el sujeto convencido de la participación divina en los hechos del mundo y perteneciente a un contexto con prácticas religiosas fuertemente instituidas, no se invisibiliza el papel de los traumatismos, las enfermedades o el ciclo vital (Albarghouthi, 2012; Wagner et al., 2000). En el caso de nuestros niños, estas mismas coordinaciones o correspondencias inestables entre observables biológicos y motivaciones de otro orden, son típicas en las referencias al MRO.

\section{Argumentos acerca del MDM}

Algo diferente constatamos respecto del segundo momento que distinguimos, referido al después de la muerte (MDM). Las nociones infantiles espontáneas sobre este más allá se encuentran menos orientadas por los observables del cuerpo y los conocimientos biológicos en general. La cuestión del después de la muerte es, para los niños, un período de levantamiento del imperio de las invariantes físicas admitidas y de los principios de la biología. En este sentido, las excepciones que allí entran en vigencia no se aplican solamente a las almas, espíritus u otras entidades asociadas al cuerpo, como podría esperarse. Por ejemplo, los niños que no realizan un desdoblamiento del ser de una persona y creen que lo que se va al cielo después de la muerte es "la persona entera" -con su ropa y hasta con algunas pertenencias-, también comienzan a aceptar, al referirse a este MDM, ideas que no aceptaban antes, al esgrimir las razones de ocurrencia de la muerte (MRO). Entre esas ideas, una de las más notorias es la de la transparencia, que permite atravesar objetos o que dos cuerpos ocupen simultáneamente el

8 Entrevista clínico-crítica administrada en la ciudad de La Plata, Argentina, en mayo de 2013. 
mismo lugar, algo imposible para un niño desde las primeras adquisiciones sensoriomotrices con las que los objetos físicos y el espacio se organizan. Es así que, usualmente, los niños atribuyen a los muertos capacidades como la flotación y la posibilidad de volar, la percepción desde el cielo de todo lo que ocurre en la tierra, la "lectura de pensamientos" de los vivos o bien ciertos poderes mágicos como el de la curación a distancia.

La nueva lógica que comienza en el MDM se encuentra, del mismo modo, en las ideas de los niños de contextos seculares. Lo notable es que en estos grupos están igualmente presentes las diferentes versiones del cielo, el paraíso, las nubes, entre otras representaciones religiosas del lugar de los muertos -imágenes que se reproducen en los medios de comunicación y entretenimiento de la cultura de masas, no sólo en los discursos religiosos (Bottini, 2014; Dennis, 2009; Ordal, 1983)-. Por lo tanto, es posible encontrar rastros de esta nueva legalidad del cuerpo en argumentos infantiles diferentes. Por ejemplo, Mariano $(5,1)$ recuerda que en una oportunidad caminó junto a sus padres por la zona de un accidente ferroviario, donde una persona murió arrollada. Al llegar al lugar el accidente ya había ocurrido y sólo quedaban allí los bomberos encargados de lavar el derrame de combustible del suelo. Al ver las burbujas de detergente en el piso, Mariano inmediatamente pensó que la persona muerta se había convertido en espuma ${ }^{9}$, aunque, al preguntarle sobre este cambio, niega rotundamente que una persona viva pudiera sufrir una transformación semejante. Del mismo modo, sostiene que es imposible que la persona vuelva a vivir desde el estado de burbujas, quedando así reservada esta nueva existencia a un MDM irreversible ${ }^{10}$.

De todas formas, si bien las referencias al MDM dan cuenta de la entrada en vigencia de una nueva legalidad del cuerpo y de la experiencia física, existen correspondencias débiles entre los observables del cuerpo y las representaciones sobre el más allá de las que el niño se apropia a partir de los relatos, imágenes y prácticas de su grupo familiar. Al respecto, este fragmento de la entrevista a Javier $(5,5)$ es elocuente:

- ¿Para qué se fue al cielo? ¿Por qué no se quedó ahí abajo? [la persona muerta de su dibujo] -Y, no sé. - ¿Por qué será? -Para eso necesitamos un libro del cuerpo humano, el abuelo lo tiene. - ¿Necesitamos eso para saber? - Si. ${ }^{11}$

Javier está absolutamente seguro de la partida al cielo de la persona muerta - una creencia que frecuentemente es muy temprana y aún previa a la comprensión de los aspectos más fundamentales de la muerte, como la irreversibilidad y la universalidad-. Sin embargo, no puede hallar las razones de la necesidad de esa partida en la misma representación inculcada y

9 Aunque no lo indagamos durante la entrevista, creemos que la idea de la transformación en espuma puede tener origen en el famoso cuento infantil La sirenita, escrito por Hans Christian Andersen, reeditado y adaptado para cine y televisión en varias oportunidades. Allí, la sirenita le pregunta a su abuela por la finitud de la vida, quien le responde que cuando las sirenas mueren se convierten en espuma de mar. Si esta hipótesis es cierta, se trata de un caso ejemplar de interferencia entre representaciones seculares no biológicos y conocimiento de la muerte.

10 Entrevista clínico-crítica administrada en la ciudad de La Plata, Argentina, en noviembre de 2013.

11 Entrevistas clínico-críticas administradas en la ciudad de La Plata, Argentina, en mayo de 2012. 
cree que deben buscarse en el terreno de una biología que comprende parcialmente. Cuando no es ignorada, esta laguna en el conocimiento sobre la relocalización en el cielo del fallecido, orienta las exploraciones de los niños en sentidos diversos. En el caso de Javier, la búsqueda de razones dirigida hacia el dominio biológico da cuenta de la correspondencia establecida entre dominios diferentes, así como, en otros niños, las razones de la relocalización se hallan en la voluntad de Dios - "los ángeles lo vienen a buscar y lo levantan" - o en el campo de fenómenos propios del mundo físico -"sube porque cuando se muere es más liviano".

Ahora bien, ¿en qué sentido un conjunto de creencias débilmente articuladas con observables y conocimientos del cuerpo puede considerarse una explicación, sensu stricto, de la muerte? Una explicación, entendida como una búsqueda de razones para dar cuenta de un fenómeno, supone un proceso deductivo. Al mismo tiempo, debemos reconocer que "buscar la razón o la explicación es admitir implícitamente la insuficiencia de un simple reduccionismo" a una ley general (Piaget, 1977, p. 11). La paradoja de la explicación, entonces, es la de la coordinación entre lo contingente y lo necesario; "se trata, por una parte, de conciliar la necesidad con la producción de cambios y, por otra, con la construcción de novedades" (Ibidem, p. 12). De este modo, los niños "explican" la muerte, en sentido amplio, mediante argumentos que se orientan privilegiadamente hacia alguno de los dos momentos, MRO y MDM. Estos argumentos no siempre constituyen deducciones apoyadas en conceptos, sino relaciones y correspondencias simples entre conocimientos de diferente naturaleza que, de manera arbitraria, se erigen en el lugar de las premisas generales o particulares de los juicios transductivos (Tau y Lenzi, 2016; Piaget, 1928). A su vez, las explicaciones infantiles de la muerte son el testimonio de la modulación que operan las representaciones sociales, las creencias, las prácticas y los valores del grupo social sobre el conocimiento infantil de tipo físico y biológico. En el seno de estas explicaciones distinguimos dos ejes fundamentales que caracterizan a la noción de muerte: el del espacio y el del tiempo.

\section{La topología de la muerte}

De las muchas transformaciones observadas en el desarrollo de la comprensión de la muerte, hay una que resulta particularmente interesante porque permite poner en evidencia la relación entre las nociones infantiles más desarrolladas y sus precursores. Nos referimos a la progresiva comprensión de la finitud de la vida y la manera en la que esta idea es asimilada, inicialmente, a ciertos esquemas topológicos.

Ahora bien, nadie dudaría del desafío psíquico que implica, en términos afectivos, el establecimiento de una representación de la muerte o extinción, ni de la relación problemática que ello guarda con la identidad subjetiva y social como instancias historizadoras y garantes de los intercambios sociales. Contrariamente, entre los investigadores no hay acuerdos equivalentes en el terreno cognitivo. ¿Por qué la comprensión de la muerte resulta un desafío 
cognoscitivo relevante? Las respuestas a este problema se orientan en diferentes direcciones. Una de las más comentadas refiere al hecho de que la propia noción de muerte no parece ser un concepto o idea homogénea, sino un paquete conceptual del que participan diversas subnociones con desarrollos independientes y no sincronizados (Harris, 2011; Lazar y Torney-Purta, 1991; Speece, 1995; Vlok y Witt, 2012). Sin negar la tesis de los subcomponentes nocionales, creemos que hay otro aspecto relevante que no ha despertado suficiente atención en la bibliografía especializada: la frecuente caracterización de la finitud de la vida en términos espaciales o como persistencia de la existencia. ${ }^{12}$ Identificamos, de esta manera, a todas las representaciones de la muerte en términos de permanencia de algún tipo de actividad, de una nueva vida, o de la suspensión sólo parcial de las manifestaciones vitales, por oposición a una detención o eliminación absoluta de las acciones de las personas muertas (Tau y Lenzi, 2015). Esta persistencia se realiza siempre en un lugar, lo que nos conduce a la constante espacialización constatada en los argumentos infantiles.

Denominamos espacialización a la comprensión de la muerte, no en términos de los procesos biológicos o de las representaciones religiosas usuales, sino de un lugar. Consideramos, entonces, que los niños "espacializan" la muerte cuando la caracterizan primordialmente por un locus o espacio post mortem en el que se sitúa el cuerpo o cualquier otro ente asociado reconocido -alma, espíritu, persona, ángel, recuerdos, etc.-. Entendida de este modo, la muerte no es un estado, un acontecimiento biológico o una finalización de las funciones vitales, sino una ubicación que se corresponde con el MDM. Estar muerto es situarse en un lugar específico, sin que esa relocalización suponga, necesariamente, una pérdida de las capacidades que se poseía en vida. Las relocalizaciones usuales de nuestros niños pertenecientes a contextos familiares creyentes son: el cielo, el paraíso, el infierno, la casa de Dios, las nubes o las estrellas. Estos lugares no se asocian con una idea de muerte entendida como cesación de funciones biológicas, sino que constituyen la misma definición de la muerte. En los niños más pequeños, que todavía no han construido indicadores y observables claros para distinguir a una persona dormida de una muerta, por ejemplo, es la localización el aspecto definitorio. De hecho, para estos niños es la ubicación en esos espacios particulares la prueba de que alguien está muerto, ya que, respecto de cualquier otra actividad, posiblemente no se admita diferencia alguna.

De todos modos, esta caracterización de la muerte por relocalización no parece ser el resultado exclusivo de las imágenes religiosas del más allá. De hecho, en los niños pertenecientes a familias ateas o agnósticas la relocalización es igualmente frecuente, aunque refiere a otros espacios diferentes de los del grupo creyente. En este último caso distinguimos una serie de representaciones espacializadas de la muerte que toman por locus definitorio a los cementerios, los ataúdes, la tierra, las camas o los hospitales. Se trata siempre de un tipo de espacio con propiedades terrenales, porque no se observa en él un levantamiento de los principios de la experiencia física y biológica. Asimismo, este tipo de espacio comparte con el cielo u otros

12 Identificamos, de esta manera, a todas las representaciones de la muerte en términos de permanencia de algún tipo de actividad, de una nueva vida o de la suspensión sólo parcial de las manifestaciones vitales, por oposición a una detención o eliminación absoluta de las acciones de las personas muertas. 
lugares distintivos de las representaciones de los grupos creyentes, el hecho de constituir un espacio-noción, una topología que en sí misma se ofrece como caracterización del fenómeno de la muerte. Por ejemplo, Lía $(6,1)$, al explicar su dibujo de una nena en una cama nos dice:

- Es una cama con una nena muerta. - ¿Y cómo sabés que la nena está muerta? -¿Cómo sé? Porque como está en la cama es que está muerta. -Ah, ¿si no estuviera en la cama no estaría muerta? -No. - ¿Y en la cama qué hace? ¿Puede ver? Puede ver, pero poquito. [Anteriormente Lía había señalado que la diferencia entre la actividad de una persona viva y la de una persona muerta es de grado y no de tipo: un muerto ve, escucha o respira poquito, un vivo, mucho]. ${ }^{13}$

La espacialización parece cumplir una función de precursor cognitivo de la comprensión de la transformación irreversible que supone toda muerte. Y por el hecho de ser anterior a la comprensión de la cesación de las funciones vitales y a la diferenciación de los observables orgánicos, los niños que admiten la relocalización como condición para la ocurrencia de la muerte, pueden, o bien asegurar que las personas muertas continúan realizando las mismas actividades propias de cualquier persona viva, o bien negarlas, total o parcialmente, si es que la noción de muerte es asimilada a la inactividad cinética. No obstante, cualquiera de estas dos posiciones contrarias reclama la existencia de un espacio en el cual el muerto alcanza su estatuto fundamental. Los siguientes fragmentos de entrevistas ilustran las dos posiciones:

Ofelia $(6,5):-¿$ Me contás qué es lo que dibujaste? -Esta persona se murió y lo llevó el rayo para el cielo. - ¿Cómo nos damos cuenta en el dibujo que está muerta la persona? -Porque una gente lo lleva hasta ahí para estar cerca de una nube [...] lo lleva un rayo de sol. - ¿Un rayo de sol se lo lleva para el cielo? -Sí. - ¿Y qué puede hacer en el cielo? -No hace nada, nada. - ¿Cómo es el cielo?; ¿qué hay ahí? -Nubes. - ¿Algo más? -Otros animales... o personas. - ¿Vivas o muertas? -Muertas. $-¿ Y$ en el cielo las personas comen? -No. - ¿Toman agua? -No. -¿Qué hacen ahí? -Nada. - ¿Charlan? -No, si una gente está muerta no puede charlar ni pensar en la nube. - ¿Y puede mirar? -No.

Paula (5, 5): -Esta persona del dibujo que está en el cielo, ¿está viva o está muerta? -Muerta, ¿no ves que está en el cielo? -Y cuando está muerta, en el cielo, ¿puede hablar? -No. - ¿Y puede escuchar? -Sí. Ah, escuchar sí... - Un poquito. - ¿Y puede correr y jugar? -Sí, puede jugar o puede correr así [imita la acción]. ${ }^{14}$

El primer ejemplo da cuenta de una espacialización de la muerte sin actividad física o mental asociada. En el espacio que le corresponde al MDM todas las funciones se suspenden, aunque la persona sigue existiendo en el lugar donde habitan y se definen los muertos y la

13 Entrevista clínico-crítica administrada en la ciudad de La Plata, Argentina, en marzo de 2012.

14 Entrevista clínico-crítica administrada en la ciudad de La Plata, Argentina, en febrero de 2012. 
muerte. El segundo fragmento muestra que la relocalización constituye una suerte de reedición de la vida, aunque con algunas limitaciones o disminuciones en las capacidades de la persona.

Se advierte en estos casos que la muerte es estar - antes que ser- de un modo nuevo, acontecido siempre en el MDM. El espacio que define a la muerte comienza siendo un observable por oposición a la noción de vida -es decir, un factor de motilidad-y por esta razón para muchos niños el espacio de la muerte es el mismo lugar que habitan quienes todavía no han nacido, es decir, no viven aún. Así, el cielo, las nubes o las estrellas, al igual que los hospitales o ciertas ciudades lejanas, son una suerte de depósito de bebés que aguardan el momento para nacer, una vez más, gracias a una relocalización.

\section{La secuencia temporal en la comprensión de la muerte}

La indistinción infantil entre los observables del MRO, que más tarde en el desarrollo serán elevados al estatuto de causas o efectos diferenciados, por un lado, y la noción de muerte (MRO + MDM), por otro, es algo que se constata en la identificación de la muerte con un lugar, con un sentimiento, con la enfermedad, con un afecto o emoción en los deudos, con la maldad o con un trauma. Esta indiferenciación es correlativa a la escasa coordinación entre el conocimiento biológico y las primeras ideas sobre el final de la vida. En el seno de este sincretismo infantil encontramos una particular organización temporal de los acontecimientos de los dos momentos a los que nos hemos referido (MRO y MDM).

Respecto de la secuencia con la que se organizan los hechos a la hora de justificar o explicar la muerte (MRO), los niños no ofrecen inicialmente argumentos lineales, de único sentido y temporalmente irreversibles, como los que es posible hallar en los discursos seculares o religiosos de los adultos. Por el contrario, los primeros suelen caracterizar la muerte de manera egocéntrica, como un acontecimiento del que no necesariamente participa la persona fallecida, aunque sí el propio niño: “¿Cómo podemos hacer para darnos cuenta de que una persona se murió?" "Porque nos llaman por teléfono y nos avisan" (Sofía, 6, 6) 15; "No lo vemos más" (Pablo, 5, 0)16; "Nos avisan en el hospital" (José, 7, 11)17; "Vas a visitarlo y no está más" (Laura 5, $1)^{18}$.

No hay aquí circularidad o inversión del orden entre el MRO y el MDM sino indistinción, coordinaciones inestables y sincretismo. Pese a que no hay circularidad en las relaciones establecidas entre ambos momentos -aunque sí son circulares los argumentos ofrecidos por los niños-, el resultado de esta indiferenciación es la distorsión de la flecha del tiempo que caracteriza al pensamiento adulto.

Por otra parte, la secuenciación infantil inicial de los momentos de vida y muerte, que

15 Entrevista clínico-crítica administrada en la ciudad de La Plata, Argentina, en junio de 2012.

16 Entrevista clínico-crítica administrada en la ciudad de La Plata, Argentina, en agosto de 2012.

17 Entrevista clínico-crítica administrada en la ciudad de La Plata, Argentina, en abril de 2012.

18 Entrevista clínico-crítica administrada en la ciudad de La Plata, Argentina, en noviembre de 2013. 
desembocarán finalmente en la caracterización del ciclo vital, dan cuenta de un tiempo reversible. Los niños más pequeños de nuestra muestra admiten que es posible volver a vivir luego de morir - mediante curaciones médicas, descensos desde el cielo, o desentierros, por ejemplo-. En este caso sí se trata de una circularidad entre dos momentos, el de la vida y el de la muerte. Este ciclo puede tener su inicio tanto en el nacimiento como en cualquiera de los puntos del circuito, como lo muestran los niños que describen el cielo como un reservorio de bebés por nacer. De este modo, el comienzo del ciclo no es el nacimiento sino una existencia previa en el espacio de los muertos, donde los bebés coexisten con las personas que ya nacieron, murieron y esperan para regresar a la vida terrenal, reeditando el ciclo.

Este tiempo circular no es remplazado luego de manera súbita al emerger la noción de irreversibilidad absoluta de la muerte -es decir, la convicción de la imposibilidad de volver a vivir una vez que se ha muerto-. En un nivel intermedio, entre ambos extremos, los niños introducen soluciones de transición, como las que denominamos "irreversibilidades por epiciclos" (ver figura 2). Allí, la irreversibilidad es parcial, porque si bien se acepta una secuencia uniforme en un único sentido, se admiten epiciclos o bucles de reversibilidad limitada dentro de la misma secuencia. Por ejemplo:

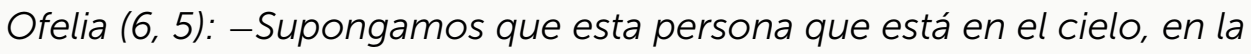
nube, y se lastima o le disparan, ¿se puede volver a morir? -Sí. - ¿Y qué le pasa? -No sé... se va a otra nube. - ¿Y si en la otra nube la vuelven a matar? -Se va más arriba. - Y si a la persona la matan en el cielo, acá en esta nube, ¿la pueden volver a enterrar? -Sí. - ¿Cómo hacen? - ¿Para bajar?... No, cuando está en la nube ya no puede bajar más. - ¿La pueden enterrar si le disparan en la nube? -No, porque la nube es suave. ${ }^{19}$

Lía $(6,1)$ : - ¿Las personas que están en el cielo, se pueden volver a morir? -No. - ¿Cómo es eso? -Algunas personas se mueren otra vez. $Y$ cuando ya están muertas, todavía se quedan en el cielo... -A ver, explícame -Cuando están en el cielo, y se enferman, se mudan a otra nube. Entonces es cuando están mejor las personas. ${ }^{20}$

Figura 2. Reversibilidad-irreversibilidad infantil de la muerte.

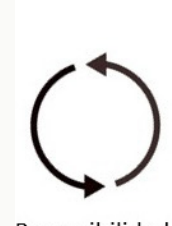

Reversibilidad

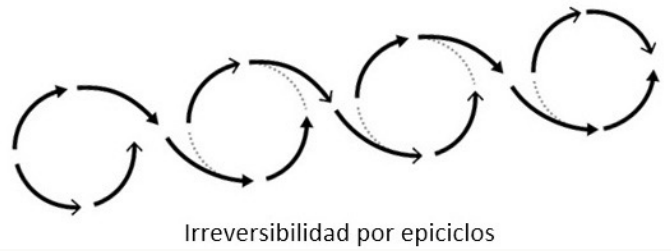

Irreversibilidad por epiciclos

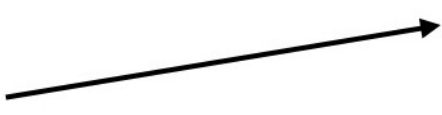

Irreversibilidad absoluta

19 Entrevista clínico-crítica administrada en la ciudad de La Plata, Argentina, en junio de 2012.

20 Entrevista clínico-crítica administrada en la ciudad de La Plata, Argentina, en marzo de 2012. 
La adquisición de la irreversibilidad por parte de los niños no debe confundirse con la de la irretornabilidad. Mientras que la primera refiere a la imposibilidad de volver a vivir, la segunda consiste en la imposibilidad, del cuerpo, la persona o el alma, de regresar a su lugar de vida previa, al mundo de los vivos o, de manera genérica, a la tierra, aun admitiendo que la vida se reedite en otro lugar, total o parcialmente. Consideramos que estas dos formas de no retorno se diferencian gradualmente y muestran de qué modo tiempo y espacio nacen indiscriminados en la comprensión de la muerte y progresivamente se van diferenciando. A partir del conjunto de los datos obtenidos, establecimos que, en un primer nivel de reversibilidad, el regreso a la vida implica el retorno al lugar previo a la muerte. En un segundo nivel, la reversibilidad es posible, pero no la retornabilidad. Dicho de otro modo, se admite que la vida es reeditable, aunque nunca en el mismo lugar en el que había ocurrido la vida previa. Finalmente, la irreversibilidad absoluta implica una distinción completa entre las dos formas de no retorno y la subsunción de ambas en un sistema temporal de transformaciones irreversibles. En los dos primeros niveles el tiempo se encuentra definido por el espacio. En el último, la secuencia temporal se desancla del espacio.

\section{Del sincretismo a la polifasia: nuevos problemas}

Tanto la espacialización como la organización de una secuencia temporal parecen ser dos componentes centrales de las ideas de los niños sobre la muerte. Según nuestra hipótesis, son estas primeras nociones sobre el tema las que dan lugar a las nociones polifásicas de los adultos que se reencuentran en prácticas y creencias individuales e instituidas (Moscovici, Jovchelovitch y Wagoner, 2013; Wagner et al., 2000).

Esas nociones se producen a través de procesos de diferenciación e integración graduales en los que no hay eliminación de la contradicción o progresión hacia una racionalidad única, sino constitución de diferentes vías o lógicas. Las diferentes creencias y prácticas culturales en torno a la muerte son elocuentes respecto del carácter variable que pueden adoptar las representaciones sobre este tema. Mucho más desafiante, desde el punto de vista teórico, resulta constatar que la racionalidad científica, las representaciones sociales y el desarrollo del conocimiento individual acerca de los dos momentos MRO y MDM no progresan juntos hacían un conocimiento coherente y sistematizado dentro de un mismo dominio. Tampoco parece existir una orientación o teleología general y estable, impuesta desde el mundo hacia los conocimientos sobre la muerte, ya que los desarrollos en el dominio de la biología, tanto a nivel individual como institucional, se intersectan con representaciones y valores de los que no pueden señalarse niveles o estadios de complejización creciente.

Creemos que la forma de alcanzar respuestas más seguras frente a estos interrogantes es analizar la génesis de los conocimientos y representaciones, tanto individuales como sociales, reconociendo los precursores y los mecanismos intervinientes en la producción de esos 
conocimientos. Pero esta empresa es insuficiente si no se juzgan las conclusiones psicológicas a la luz del conocimiento histórico y antropológico. En este artículo intentamos señalar brevemente que la espacialización, la secuencia temporal, la replicación y la persistencia de la existencia parecen ser núcleos fundamentales del pensamiento infantil. Y es posible que estos mismos núcleos sean una parte fundamental del pensamiento de las grandes religiones (León Azcárate, 2008) y del sentido común de todos los hombres que, tarde o temprano, se enfrentan a la finitud de su propia existencia. Aquí no podemos más que plantear este interrogante que exige nuevas investigaciones específicas y un gran esfuerzo intelectual destinado a poner en discusión los hallazgos de las diferentes disciplinas interesadas en el fenómeno biológico y social de la muerte.

\section{Referencias bibliográficas}

ACADEMIA ARGENTINA DE LETRAS. Diccionario argentino de dudas idiomáticas. Buenos Aires: Santillana. 2011. 528p.

ALBARGHOUTHI, Samar Issa Zayed. The Palestinians Understanding of Cancer: A methodological perspective. The 14th conference on Social and Community psychology. Trondheim: Norwegian University of Science and Technology (NTNU), 2012.

ARRIZABALAGA, Ángel Rivera. Conducta simbólica: la muerte en el Musteriense y MSA. Zephyrvs, v. 65, p. 39-63, 14 jul. 2010.

BINANT, Pascale. La Préhistoire de la mort: les premières sépultures en Europe. Paris: Editions Errance, 1991. 168p.

BOTTINI, Maria Emília. No cinema e na vida: a difícil arte de aprender a morrer. Tese (Doutorado em Educação), Programa de Pós-Graduação em Educação, Universidade de Brasília, Brasília, 2014. 195p. Disponible en: <http://repositorio.unb.br/handle/10482/17393>. Acceso en: 20/07/2016.

CASTORINA, José Antonio. El impacto de las representaciones sociales en la psicología de los conocimientos sociales: problemas y perspectivas. Cadernos de Pesquisa, v. 38, n. 135, p. 757-776, dez 2008.

Psicología de los conocimientos sociales en los niños y teoría de las representaciones sociales. In: CARRETERO, Mario; CASTORINA, José Antonio (Eds). La construcción del conocimiento histórico. Buenos Aires: Paidós, p. 153-172, 2010.

; AISEMBERG, Beatriz; URE, Celia D.; COLINVAUX, Dominique; PALAU, Gladys. Problemas en Psicología Genética. Buenos Aires: Miño y Dávila, 1989. 234p.

; BARREIRO, Alicia; CLEMENTE, Fernando. El conocimiento de los niños sobre la sociedad según el constructivismo y la teoría de las representaciones sociales. In: CASTORINA, José Antonio (Coord.). Construcción conceptual y representaciones sociales. El conocimiento de la sociedad. Buenos Aires-Madrid: Miño y Dávila, p. 177-204, 2005. 
; FAIGENBAUM, Gustavo. Restricciones y conocimiento de dominio: hacia una diversidad de enfoques. In: CASTORINA, José Antonio; LENZI, Aicia María (comp.). La formación de los conocimientos sociales en los niños. Investigaciones psicológicas y perspectivas educativas. Barcelona: Gedisa, p. 155-180, 2000.

; KOHEN KOHEN, Raquel C.; ZERBINO, Mario C. Reflexiones sobre la "especificidad" de un subdominio del conocimiento social. In: CASTORINA, José Antonio; LENZI, Alicia María (comp.). La formación de los conocimientos sociales en los niños. Investigaciones psicológicas y perspectivas educativas. Barcelona: Gedisa, 2000, p. 135-154.

; LENZI, Alicia María; FERNÁNDEZ, Susana. Alcances del método de exploración crítica en psicología genética. In: CASTORINA, José Antonio et al. Psicología Genética. Aspectos metodológicos e implicancias pedagógicas. Buenos Aires: Miño y Dávila, p. 83-118, 1984.

DEFLEUR, Alban. Les sépultures moustériennes. Paris: C.N.R.S. Éditions, 1993. 325p.

DENNIS, Dixie. Living, Dying, Grieving. Boston: Jones \& Bartlett Learning, 2009. 246p.

GARCÍA, Rolando. El conocimiento en construcción. De las formulaciones de Jean Piaget a la teoría de sistemas complejos. México: Siglo XXI, 2000. 252p.

Fundamentación de una epistemología en las ciencias sociales. Estudios Sociologicos, v. 19, n. 3, p. 615-620, 2001. Disponible en: <http://www.redalyc.org/articulo.oa? id=59805701>. Acceso en: 17/08/2016.

GIRE, James. How Death Imitates Life: Cultural Influences on Conceptions of Death and Dying. Online Readings in Psychology and Culture, v. 6, n. 2, p. 3-22, 1 Dez 2014. Disponible en: $<$ http://scholarworks.gvsu.edu/orpc/vol6/iss2/3>. Acceso en: 24/10/2016.

HARRIS, Paul L. Conflicting Thoughts about Death. Human Development. v. 54, n. 3, p. $160-$ 168, 2011.

HONEY, Margaret A. The Interview as Text: Hermeneutics Considered as a Model for Analyzing the Clinically Informed Research Interview. Human Development. v. 30, n. 2, p. 69-82, 1987.

INAGAKI, Kayoko; HATANO, Giyoo. Young Children's Conception of the Biological World. Current Directions in Psychological Science, v. 15, n. 4, p. 177-181, Ago. 2006.

JODELET, Denise. La representación social: fenómeno concepto y teoría. In: MOSCOVICI, Serge. Psicología Social, II. Barcelona: Paidós, p. 469-494, 1986.

LAZAR, Alice; TORNEY-PURTA, Judith. The development of the subconcepts of death in young children: a short-term longitudinal study. Child Development, PMID: 1786718, v. 62, n. 6, p. 1321-1333, Dez. 1991.

LENZI, Alicia María; CASTORINA, José Antonio. Algunas reflexiones sobre una investigación psicogenética en conocimientos sociales: la noción de autoridad escolar. In: CASTORINA, José Antonio; LENZI, Alicia, María (comp.). La formación de los conocimientos sociales en los niños. Investigaciones psicológicas y perspectivas educativas. Barcelona: Gedisa, p. 41-58, 2000.

LEÓN AZCÁRATE, Juan Luis. La muerte y su imaginario en la historia de las religiones. Bilbao: Universidad de Deusto, 2008. 503p.

LIGHTFOOT, Cynthia; VALSINER, Jaan. Parental belief systems under the influence: Social 
guidance of the construction of personal cultures. In: SIGEL, Irving E.; MCGILLICUDDYDELISI, Ann V.; GOODNOW, Jacqueline J. (Org.). Parental belief systems: The psychological consequences for children. Hillsdale, NJ, US: Lawrence Erlbaum Associates, Inc, 1992, p. 393414.

LLOYD, Barbara; DUVEEN, Gerard. A semiotic analysis of the development of social representations of gender. In: DUVEEN, Gerard; LLOYD, Barbara (Eds.). Social Representations and the Development of Knowledge. Cambridge: Cambridge University Press, 1990. 191p.

MORIN, Edgar. El hombre y la muerte. Barcelona: Editorial Kairós, 1994. 378p.

MOSCOVICl, Serge. El psicoanálisis su público y su imagen. Buenos Aires: Huemul, 1979 [1961]. $363 p$.

JOVCHELOVITCH, Sandra; WAGONER, Brady. Development as a Social Process: Contributions of Gerard Duveen. London-New York: Routledge, 2013. 232p.

ORDAL, Carol C. Death as Seen in Books Suitable for Young Children. OMEGA--Journal of Death and Dying, v. 14, n. 3, p. 249-277, 1 Jan. 1983.

PFEIFFLE, Horst. On the psychogenesis of the a priori: Jean Piaget's critique of Kant. Philosophy \& Social Criticism, v. 34, n. 5, p. 487-498, 1 Jun. 2008.

PIAGET, Jean y GARCÍA, Rolando. Psicogénesis e historia de la ciencia. México: Siglo XXI, 1982. $264 p$.

Judgment and reasoning in the child. United Kingdom: K. Paul, Trench, Trubner \& Co. Itd., 1928 [1924]. 278p.

El nacimiento de la inteligencia en el niño. México: Grijalbo, 1936. 398p.

Programme et méthodes de l'Épistémologie génétique. Introduction. In: BETH, Evert Willem; MAYS, Wolfe; PIAGET, Jean. Épistémologie génétique et researche psychologique. Paris: Presses Universitaires de France, p. 13-84, 1957.

Introducción: Los problemas y los métodos. In: La Representación del Mundo en el Niño. Madrid: Morata, p. 11-40, 1975 [1926].

La explicación en las ciencias. Barcelona: Ediciones Martínez Roca, 1977 [1973]. 222p.

Psicología y epistemología. Buenos Aires: Emecé, 1979 [1972]. 141p.

La equilibración de las estructuras cognitivas: problema central del desarrollo. México: Siglo XXI, 1998 [1975]. 203p.

PSALTIS, Charis; DUVEEN, Gerard. Social relations and cognitive development: the influence of conversation type and representations of gender. European Journal of Social Psychology, v. 36, n. 3, p. 407-430, may. 2006.

PERRET-CLERMONT, Anne-Nelly. The Social and the Psychological: Structure and Context in Intellectual Development. Human Development, v. 52, n. 5, p. 291-312, 2009.

REAL ACADEMIA ESPAÑOLA. Diccionario de la lengua española. Avance de la vigésima tercera edición. 2013. Disponible en: <http://rae.es>. 
ROSENGREN, Karl S.; GUTIÉRREZ, Isabel T.; SCHEIN, Stevie S. Cognitive dimensions of death in context. Monographs of the Society for Research in Child Development, v. 79, n. 1, p. 62-82, mar. 2014.

SLAUGHTER, Virginia, JAAKOLA, Raquel; CAREY, Susan. Constructing a coherent theory: Children's biological understanding of life and death. In: SIEGAL, Michael; PETERSON, Candida C. (Eds.). Children's understanding of biology, health, and ethics. Cambridge: Cambridge University Press, p. 71-98, 1999.

SPEECE, Mark W. Children's Concepts of Death. Michigan Family Review, v. 1, n. 1, p. 57-69, 1995. Disponible en: <http://hdl.handle.net/2027/spo.4919087.0001.107>.

TAJFEL, Henri. Human Groups and Social Categories. Cambridge: Cambridge University Press, 1981. 371p.

TAU, Ramiro; GÓMEZ, María Florencia. La entrevista clínica en la investigación del conocimiento infantil. In: BORZI, Sonia Lilián (Coord.). El desarrollo infantil del conocimiento sobre la sociedad. Perspectivas, debates e investigaciones actuales. La Plata: EDULP, p. 63-77, 2016.

; LENZI, Alicia María. The notion of death as a knowledge and research object in developmental psychology. Schème - Revista Eletrônica de Psicologia e Epistemologia Genéticas, v. 7, n. 1, p. 47-65, 1 set. 2015.

La comprensión infantil de la muerte. In: BORZI, Sonia Lilián (comp.). El desarrollo infantil del conocimiento sobre la sociedad. Perspectivas, debates e investigaciones actuales. La Plata: EDULP, p. 122-146. 2016.

TOMASELLO, Michael. Los orígenes culturales de la cognición humana. Buenos Aires: Amorrortu, 2007. 296p.

VALSINER, Jaan. Developmental epistemology and implications for methodology. In: LERNER, Richard (Ed.). Handbook of Child Psychology, Vol. 1. Theoretical models of human development. New York: Wiley, p. 166-209, 2005.

La dialéctica en el estudio del desarrollo. In: CASTORINA, José Antonio; CARRETERO, Mario (comps.). Desarrollo cognitivo y educación [I]. Los inicios del conocimiento. Buenos Aires: Paidós, p. 137-162, 2012.

VALSINER, Jaan; WINEGAR, Lucien T. Introduction: A Cultural-Historical Context for Social "Context". In: WINEGAR, Lucien T.; VALSINER, Jaan (Eds.). Children's Development Within Social Context. Volume I: Metatheory and Theory. USA: Lawrence Erlbaum Associates, Publishers, p. 1-14, 1992.

VLOK, Milandre; WITT, Marike W. de. Naive theory of biology: the pre-school child's explanation of death. Early Child Development and Care. v. 182, n. 12, p. 1645-1659, 1 dez. 2012.

WAGNER, Wolfgang et al. I have some faith and at the same time I don't believe. Cognitive polyphasia and cultural change in India. Journal of Community \& Applied Social Psychology. v. 10, n. 4, p. 301-314, jul. 2000.

Recebido em: 29 de outubro de 2016.

Aprovado em: 08 de novembro de 2016. 